\title{
Active stabilization methods of electric power systems with constant power loads: a review
}

\author{
Mingfei WU (ه), Dylan Dah-Chuan LU
}

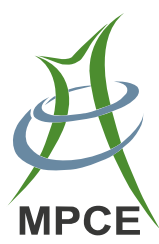

\begin{abstract}
Modern electric power systems have increased the usage of switching power converters. These tightly regulated switching power converters behave as constant power loads (CPLs). They exhibit a negative incremental impedance in small signal analysis. This negative impedance degrades the stability margin of the interaction between CPLs and their feeders, which is known as the negative impedance instability problem. The feeder can be an LC input filter or an upstream switching converter. Active damping methods are preferred for the stabilization of the system. This is due to their higher power efficiency over passive damping methods. Based on different sources of damping effect, this paper summarizes and classifies existing active damping methods into three categories. The paper further analyzes and compares the advantages and disadvantages of each active damping method.
\end{abstract}

Keywords Stabilization, LC filters, Constant power loads

\section{Introduction}

In some electric power systems, if the outputs of switching power converters are tightly regulated, the instantaneous input power of the converter is constant within the regulation bandwidth. Therefore, from the view

CrossCheck date: 14 July 2014

Received: 28 May 2014/ Accepted: 24 July 2014/Published online: 22 August 2014

(C) The Author(s) 2014. This article is published with open access at Springerlink.com

M. WU, D. D.-C. LU, School of Electrical and Information Engineering, The University of Sydney, Darlington, NSW, Australia

$(\bowtie)$ e-mail: miwu2816@uni.sydney.edu.au;

mingfei.wu@sydney.edu.au of their feeders, these tightly regulated converters behave as constant power loads (CPLs) [1-4]. CPLs have an inverse proportional $v-i$ characteristic and exhibit negative incremental impedance in small signal analysis. This negative incremental impedance can degrade the stability margin of system interaction between CPLs and their feeder system, and is known as negative impedance instability problem [5-7]. This can be illustrated by a cascaded system as shown in Fig. 1.

The upstream circuit as shown in Fig. 1 can be a passive circuit, for example, an LC filter. It can also be another power converter. The downstream circuit is a tightly regulated converter. Both upstream and downstream circuits are designed to be stable individually. i.e.; the transfer functions of the upstream circuit

$G_{A}(s)=v_{A \_o}(s) / v_{A \_}$in $(s)$

and the downstream circuit

$G_{B}(s)=v_{B \_o}(s) / v_{B} \_i n(s)$

are stable. However, the stability of the cascaded system with a transfer function of

$G_{A B}(s)=G_{A}(s) G_{B}(s) /\left(1+Z_{\text {in }}(s) / Z_{o}(s)\right)$

depends on the stability of $Z_{o}(s) /\left(Z_{i n}(s)+Z_{o}(s)\right)$. The negative incremental impedance characteristic of $Z_{i}(s)$ can degrade the stability margin of $Z_{o}(s) /\left(Z_{i}(s)+Z_{o}(s)\right)$ and consequently destabilize the operation of the cascaded system. The sufficient and necessary condition of the cascaded system stability is that the Nyquist contour of $T(s)$, as shown in (4), does not encircle the point $(-1,0)$ [8].

$T(s)=Z_{i}(s) / Z_{o}(s)$

where $T(s)$ denotes the minor loop gain of the cascaded system.

However, how to use this stability criterion for active damping design is not straightforward. Middlebrook's 


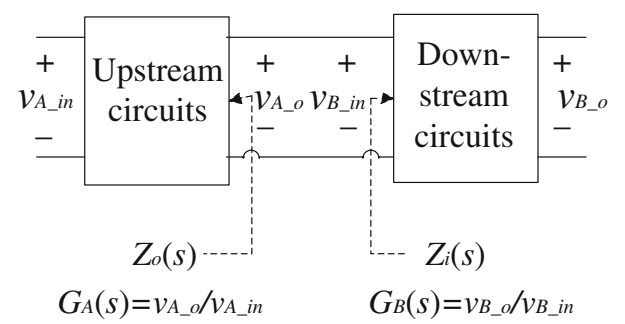

Fig. 1 The cascaded system for illustration of system stability

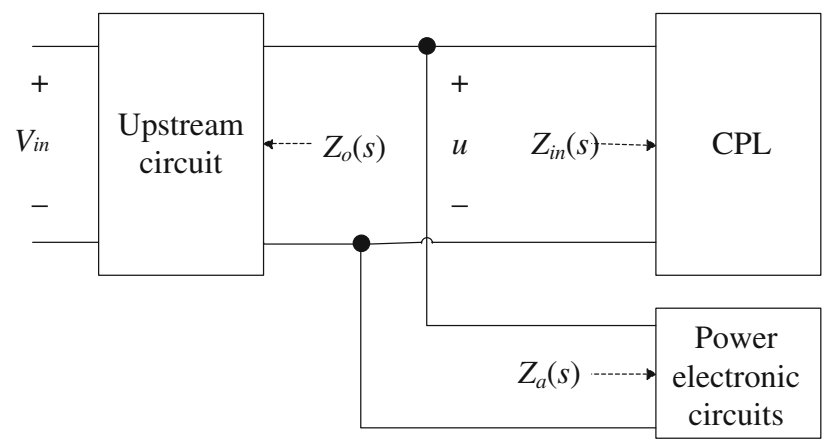

Fig. 2 Three main approaches of active damping methods

stability criterion, which is an illustrative stability criterion, has been proposed. A sufficient condition is proposed to guarantee the system stability which is shown in (5) [9].

$\left\|Z_{o}(\mathrm{j} \omega)\right\| \ll\left\|Z_{\text {in }}(\mathrm{j} \omega)\right\|$

Several similar stability criteria have also been proposed [10-12]. This paper focuses on the active methods that stabilize the cascaded system with CPL as shown in Fig. 1. The Middlebrook's stability criterion is adopted in this paper to mathematically show the effectiveness of these methods, because most of these methods are designed based on this stability criterion.

In order to stabilize the unstable system due to CPLs, several active stabilizing methods have been proposed. However, these active methods are based on different electric power system architectures and applications. In order to clarify the differences between these methods and make it easier for engineers and researchers to find a suitable method for a given system with CPLs, this paper classifies the existing methods into three categories according to different sources of stabilizing effect. Then, each method is analyzed to show its advantages and disadvantages.

The general approach of active stabilization methods is to modify $Z_{o}(s)$ or/and $Z_{i n}(s)$ to fulfill the Middlebrook's stability criterion and consequently stabilize the cascaded system. These methods can be classified into three categories according to different sources of stabilizing effect as shown in Fig. 2.

1) Modify the output impedance of the upstream circuit $Z_{o}(s)$ by increasing the bandwidth of the control loop of the upstream converter [13], or by adding an extra damping loop [14-21].

2) Modify the input impedance of the CPL, $Z_{i n}(s)$ by injecting a stabilizing current into the CPL [2, 22-28].

3) Add an auxiliary power electronic circuit between the upstream and downstream circuits and control the input impedance of the auxiliary power electronic circuit, $Z_{a}(s) . Z_{a}(s)$ can be used to modify either $Z_{o}(s)$ or $Z_{\text {in }}(s)[29,30]$.

In Sections 2, 3 and 4, these three main approaches of active damping methods are analyzed respectively. In Section 5, comparisons of these active damping methods are made and conclusion is provided.

\section{Active damping method 1: modifying the output impedance $Z_{o}(s)$ of the upstream converter}

In some electric power system configurations, the upstream circuit is a switching power converter. For example, in electric vehicles (EVs) onboard DC power systems, the feeder of CPLs is another stage of converter. This upstream converter could be an intermediate bus converter or a source converter of a distributed generator. The upstream converter could be either a DC/DC converter or an AC/DC converter. In signal analysis point of view, if the converter works in continuous conduction mode (CCM) and is in open loop control, inductor and capacitor in these source converters serve as an LC filter. This LC filter can result in a peak value around its resonant frequency in the output impedance of the source converters. Therefore, the Middlebrook's stability criterion may be violated and the system can be unstable. In addition, if the converter is in closed loop control and its bandwidth is lower than the resonant frequency, the LC filter resonant characteristic in the output impedance still exist beyond the bandwidth of the closed loop control as illustrated in Fig. 3. The relationship between the bandwidth of the closed loop control and the output impedance of the converter will be illustrated and explained in Section 2.1. Meanwhile, if the converter works in discontinuous conduction mode (DCM), zero inductor current operation changes the small signal operation of the inductor. Consequently, the output impedance of the converter may change and the cascaded system is stable. The stability analysis of DC/DC converter in DCM is presented in [31, 32].

This paper focuses on the stabilizing method of switching converters in CCM. A general approach to stabilizing this type of systems is to modify the output impedance of the source converter. One approach is to increase the closed loop control bandwidth of the source 


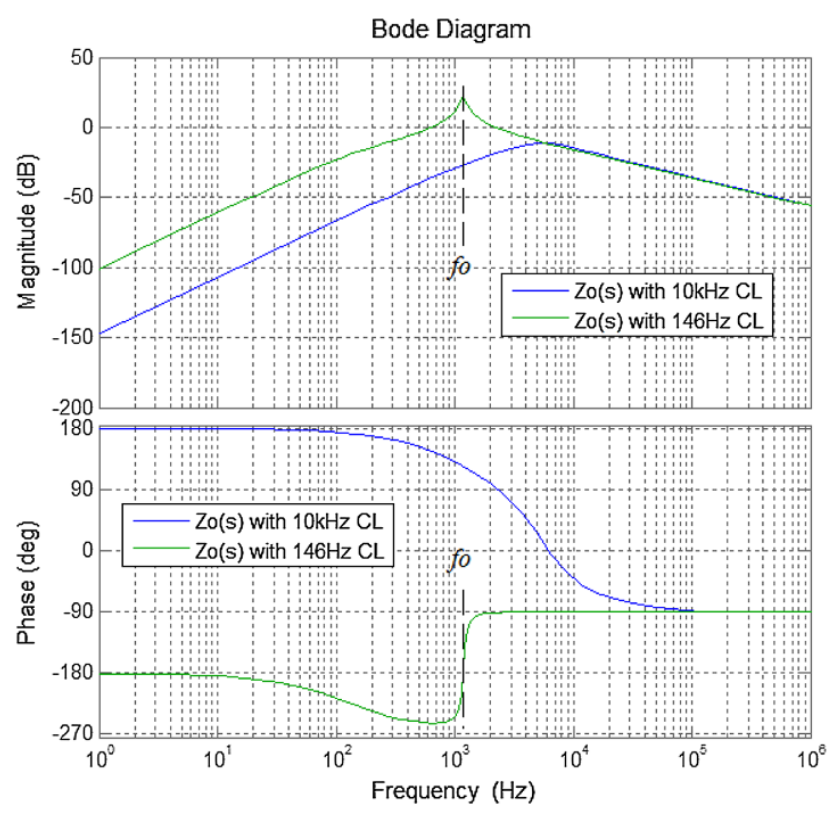

Fig. 3 The output impedance of the buck converter with different closed loop control bandwidth (CL in labels denotes the closed loop control)

converter. Another approach is to add an extra stabilizing loop.

\subsection{Increasing the closed loop control bandwidth} of the upstream converter

The upstream converter is represented by a buck converter as an example. The output impedance of the buck converter is

$Z_{o}(s)=L s /\left(L C s^{2}+V_{i n} F(s)+1\right)$

where $L$ and $C$ are inductor and capacitor of the buck converter respectively, $V_{i n}$ is the input voltage and

$F(s)=d(s) / v_{o}(s)$

is the transfer function of the voltage controller. The bode diagram of the output impedance of the buck converter with different closed loop control bandwidth is shown in Fig. 3. In this example, $L=200 \mu \mathrm{H}$ and $C=100 \mu \mathrm{F}$. The resonant frequency, $f_{o}$, of the LC filter is $1.13 \mathrm{kHz}$.

From Fig. 3, it can be found that, when the bandwidth of the closed loop control is lower than the resonant frequency of the LC filter, the output impedance of the buck converter is close to the output impedance of an LC filter. The magnitude around the resonant frequency is high, possibly causing instability operation. In contrast, when the bandwidth of the closed control loop is higher than the resonant frequency, the magnitude response is much lower. This phenomenon can be briefly explained by (6). If the cut off frequency, $f_{c}$, of closed control loop is lower than the resonance, $f_{o}$, i.e. $f_{c}<f_{o}$, where $f_{o}=1 /(2 \pi \sqrt{L C})$, the magnitude of $F\left(2 \pi f_{o}\right)$ will be much less than 1 . Thus $Z_{o}$ $\left(2 \pi f_{o}\right)$ will become large. In contrast, if $f_{c}>f_{o}$, the magnitude of $F\left(2 \pi f_{o}\right)$ will be much larger than 1 , and $Z_{o}\left(2 \pi f_{o}\right)$ will become small and it is easier to fulfill (5).

In other type of $\mathrm{DC} / \mathrm{DC}$ and $\mathrm{AC} / \mathrm{DC}$ converters, the relationship between the output impedance and the bandwidth of the closed loop control is similar to the buck converters. The peak value of the converter output impedance is large and is around the resonant frequency of the LC filter inside the converter if a low bandwidth closed loop control is applied. In this case, output impedance of the $\mathrm{DC} / \mathrm{DC}$ converter can be reduced by increasing the closed loop control bandwidth to be larger than the resonance of LC filter. However, this is usually not available for AC/DC converter. Because, in conventional control of the AC/DC converter, the control bandwidth should be designed to be lower than the line frequency, i.e. 50 or 60 $\mathrm{Hz}$, due to the need for power factor correction. In [33-37], several fast controllers for AC/DC converters have been proposed. However, the bandwidth of the closed loop control can only be increased up to around $200 \mathrm{~Hz}$ which is normally lower than the resonant frequency of the LC filter inside the converter. Consequently, the LC resonance peak in the output impedance is almost not reduced. Therefore, the stabilizing method through increasing the control bandwidth may not applicable for AC/DC converter.

Based on the above analysis, when the upstream converter is a DC/DC converter, one simple method to reduce the output impedance is to have a high closed loop control bandwidth. In [13], Du, et al have given the criteria for closed loop controller design of upstream DC/DC converter for the stability of the cascaded system with CPL.

\subsection{Adding an extra stabilizing loop}

In some applications, high closed loop control bandwidth is not available or the upstream converter works in open loop control. In this situation, an extra stabilizing loop is required for stabilization of the cascaded system. For small signal stability, linear methods are proposed. However, in some applications, large signal stability is required, or the CPL works in several operating points. In these conditions, several nonlinear methods are proposed.

\subsubsection{DC/DC converter}

Several active stabilizing methods have been proposed for DC/DC converter with CPL. One linear and effective method is to build a virtual resistor in series with the inductor in the DC/DC converter [14]. This virtual resistor 


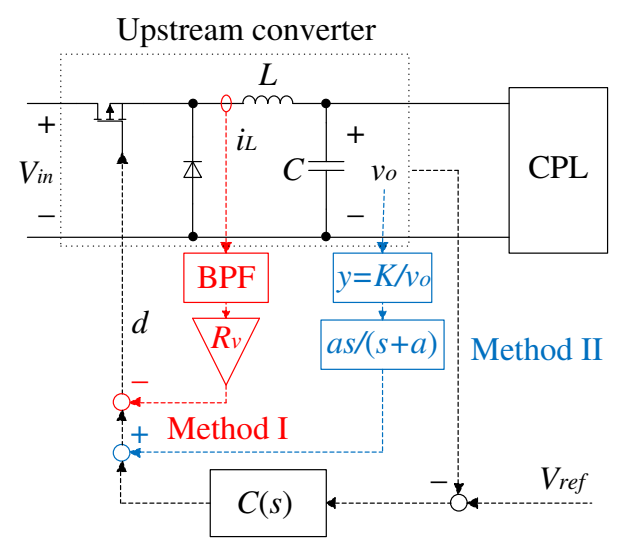

Fig. 4 The configuration of the two extra stabilizing loops for a buck converter loaded by CPL

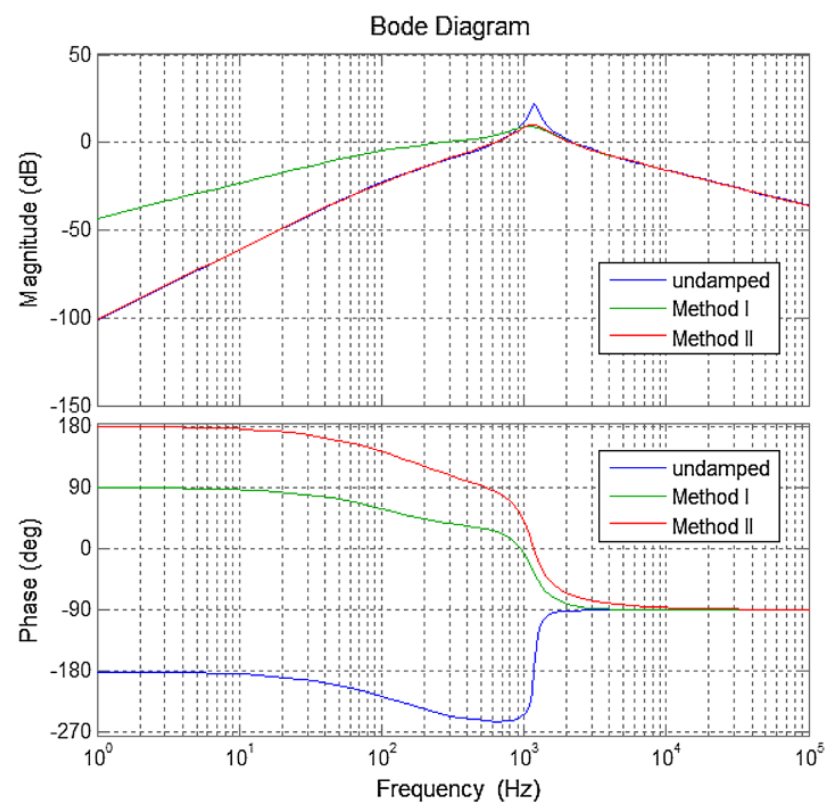

Fig. 5 Bode diagrams of the output impedances of the buck converter with the two active damping methods

reduces the peak value of the output impedance. The configuration of this stabilizing loop is shown in Fig. 4 and is named as Method I. In Fig. 4, a buck converter is used as an example. Another method is to add a nonlinear feedback loop to cancel the nonlinearity of the CPL and thus stabilize the system [15]. In small signal analysis, this method builds a virtual resistor in parallel with the CPL. Thus, the output impedance of the DC/DC converter can be reduced. The configuration of this method is illustrated in Fig. 4 and named as Method II. The bode diagram of the output impedance of the buck converter with these two methods is shown in Fig. 5.

From Fig. 5, it can be observed that both Methods I and II can effectively reduce the output impedance of the DC/
DC converter around the resonant frequency of LC filter. In Method I, the virtual resistor $R_{v}$, has to be within the small or large signal stability criteria as shown in (8) and (9) respectively [38].

$L / C R<R_{v}<R$

$\sqrt{L / C}<R_{v}<R$

where $L$ and $C$ are the inductor and capacitor of the buck converter and $R$ is the resistor of the CPL. However, if the ratio of $L / C$ is large and $R$ is small, $R_{v}$ may not exist. In contrast, in Method II, given any power level of CPL, there always exist a constant $K$ to stabilize the cascaded system. In addition, in Method I, a current sensor is used meanwhile a voltage sensor is used in Method II.

In addition, there are other nonlinear active stabilization methods. Sliding mode control is proposed for stabilization of a DC/DC buck converter with CPL. The benefits of this method is that it can stabilize the cascaded system in several operating points [18]. A passivity based control method is also proposed for a DC/DC boost converter. The main advantage of this method is that it can obtain a fast response when load condition changes [19]. A boundary control method for DC/DC converter with CPL is proposed in $[16,17]$. This method can provide faster transient, more robust operation compared with conventional PID controller. However, all these three methods require both voltage and current sensors.

\subsubsection{AC/DC converter}

The upstream converter can also be an AC/DC converter. In [20], a linear active stabilization method by modifying the output impedance of AC/DC converter is presented. Three kinds of stabilizing loop are proposed and compared. The configuration of the most effective stabilizing loop is shown in Fig. 6. The output impedance of the AC/DC converter with this stabilizing loop is shown in Fig. 7. With the stabilizing method, the output impedance of the AC/DC converter can be reduced and the Middlebrook's stability criterion can be fulfilled.

In addition, sliding mode control method is also proposed for AC/DC converters with CPLs. Sliding mode control guarantees the stability in large range of operating points [21]. However, this method requires both voltage and current sensors.

\section{Active damping method 2: modifying the input impedance $Z_{\text {in }}(s)$ of CPLs}

In some DC power electric systems, the feeder of a CPL is an LC input filter. In addition, in AC power systems, a 


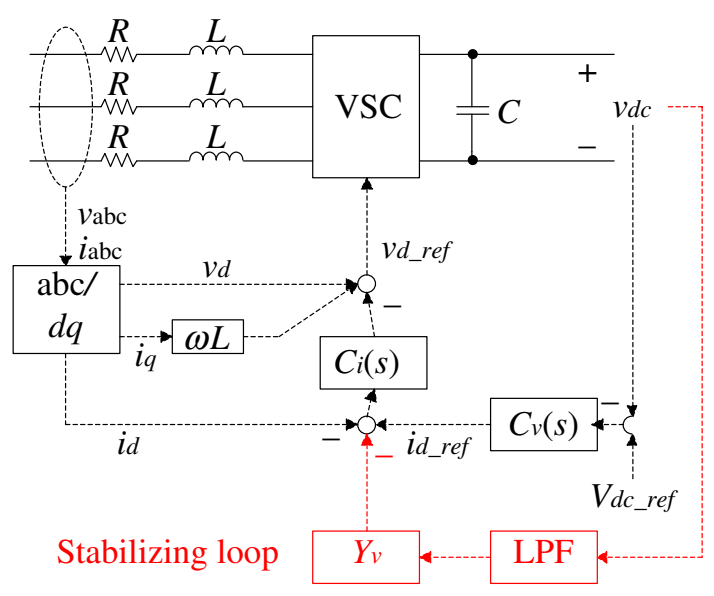

Fig. 6 The configuration of the stabilizing loop in the AC/DC converter

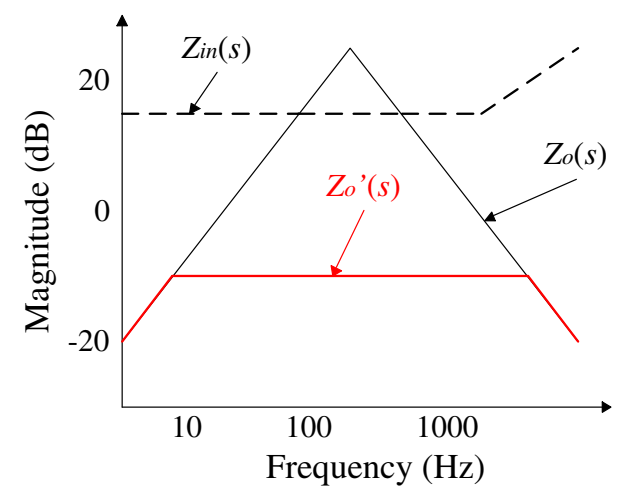

Fig. 7 The magnitude response of the output impedance of the AC/ DC converter without stabilizing method (in solid black) and with stabilizing method (in solid red)

diode type rectifier is equivalent to an LC filter as shown in Fig. 8. In Fig. 8, an AC load is used as an example but it can also be a DC load.

In these configurations, the upstream circuit is a passive LC filter. High bandwidth control of the upstream circuit is not available. Therefore, the damping effort can be only from CPLs themselves. In order to stabilize such cascaded system, some active damping methods have been proposed. These methods can be classified into linear methods and nonlinear methods. In linear methods, compensating current are injected into the CPL to modify the input impedance of CPL, $Z_{i n}(s)$, such that Middlebrook's stability criterion is fulfilled.

\subsection{Linear methods}

In linear methods, a stabilizing power is injected into the CPL to modify its input impedance. The configuration of the linear methods is shown in Fig. 9.
AC power supplies a CPL

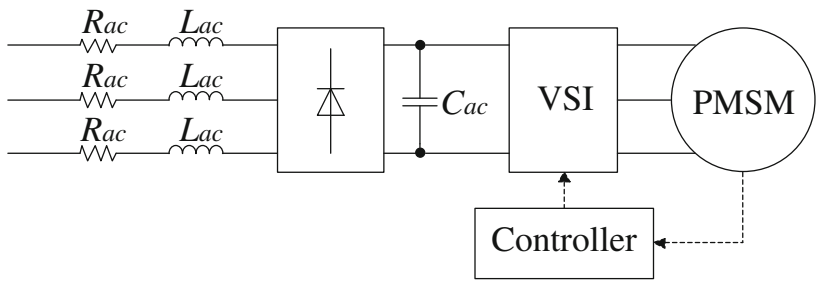

DC power supplies a CPL

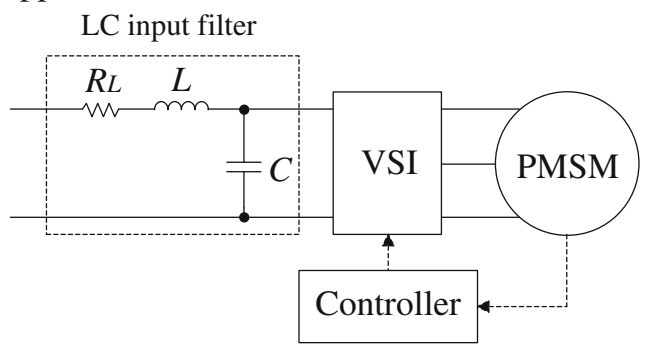

Equivalent circuit

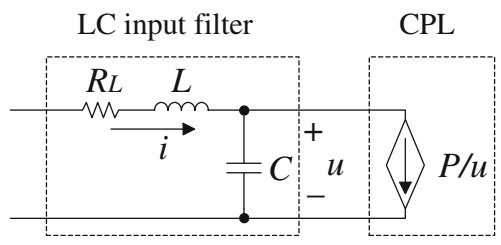

Fig. 8 A CPL in AC power system and DC power system and their equivalent circuit

The operation of the cascaded system with linear methods can be described in (10).

$\left\{\begin{array}{l}\frac{\mathrm{d} i}{\mathrm{~d} t}=-\frac{R_{L}}{L} i-\frac{1}{L} u+\frac{1}{L} V_{i n} \\ \frac{\mathrm{d} u}{\mathrm{~d} t}=\frac{1}{C} i-\frac{P+P_{\text {stab }}}{C u}\end{array}\right.$

where, $i$ and $u$ are inductor current and capacitor voltage respectively. $L$ and $C$ are the inductor and capacitor of the LC filter respectively. $R_{L}$ is the equivalent resistor of the power cable and physical resistor of the inductor. $V_{\text {in }}$ is the input voltage of the LC filter. $P$ is the power of CPL and $P_{\text {stab }}$ is the power used for stabilize the system. $P_{\text {stab }}$ is injected into the CPL to build virtual resistor or virtual capacitor parallel connected with LC filter capacitor depending on different linear methods as illustrated in Fig. 9.

There are two requirements for $P_{\text {stab }}$ [39].

1) With $P_{\text {stab }}$, the input impedance of the CPL can be modified to fulfill the Middlebrook's stability criterion.

2) In steady state operation, $P_{\text {stab }}=0$.

In the linear methods, the stabilizing power $P_{\text {stab }}$ is represented by stabilizing current, $i_{\text {stab }}$ and 


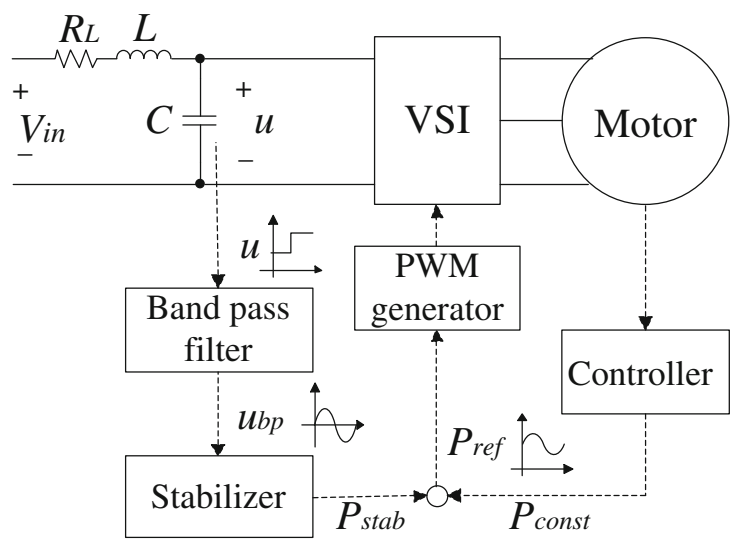

Linear stabilizing loop

Closed loop control

Equivalent circuit

LC input filter

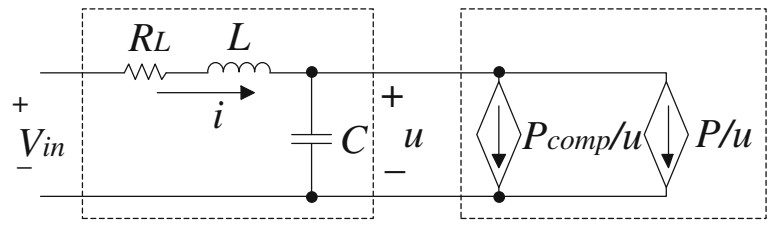

Function of these linear methods

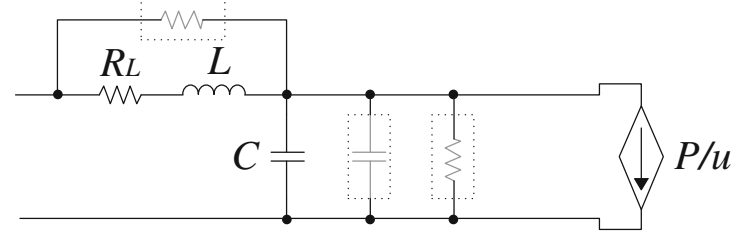

Fig. 9 The configuration of the cascaded system with linear methods

$i_{\text {stab }}=\frac{P_{\text {stab }}}{C u}$

After linearization around the operation point, (10) can be rewritten as

$\left\{\begin{array}{l}\frac{\mathrm{d} i}{\mathrm{~d} t}=-\frac{R_{L}}{L} i-\frac{1}{L} u+\frac{1}{L} V_{i n} \\ \frac{\mathrm{d} u}{\mathrm{~d} t}=\frac{1}{C} i+\frac{u}{C R}+i_{\text {stab }}\end{array}\right.$

where $R$ is the resistance of the CPL.

In order to see the effectiveness of active stabilization methods, the performances of the DC brushless motor without damping methods are shown in Fig. 10. From Fig. 10, it can be found that, during the start of the motor, when the rotating speed $\omega_{r}$ is small, $P$ is small and $R$ is large and is larger than $\left\|Z_{o}(s)\right\|$. Hence, the system is stable. However, when $\omega_{r}$ increases larger, then $R$ becomes small and is smaller than $\left\|Z_{o}(s)\right\|$, the system becomes unstable.
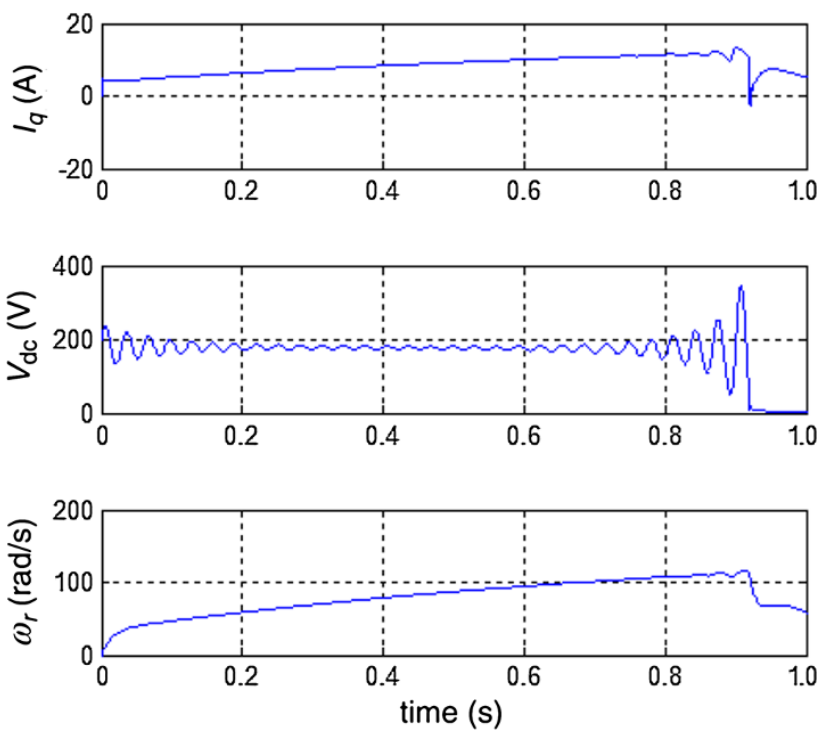

Fig. 10 The performances of the DC brushless motor without damping methods

\subsubsection{Virtual resistor $R_{v}[2,23-25]$}

Modifying (11) so that

$i_{\text {stab }}=\frac{C_{b}(s) u}{C R_{v}}$

where, $C_{b}(s)$ is the transfer function of a band pass filter with unity gain, it represents a virtual resistor $R_{v}$ that is being built in parallel with the LC filter capacitor. The function of the band pass filter is to pick the oscillation component which is around the resonant frequency of the LC filter and attenuate the steady state value and switching noises. In order to stabilize the system, $R_{v}$ needs to be chosen such that Middlebrook's stability criterion is fulfilled as shown in Fig. 11.

\subsubsection{Virtual capacitor $C_{v}[22,23]$}

When (11) is modified so that,

$i_{\text {stab }}=\frac{s C_{v} C_{l}(s) u}{C}$

where $C_{l}(s)$ is a low pass filter with unity gain, a virtual capacitor $C_{v}$ is built in parallel with the LC filter capacitor. Thus the overall capacitance of the LC filter is increased. Also, the value of $C_{v}$ needs to be chosen to make the Middlebrook's stability criterion fulfilled as shown in Fig. 12.

\subsubsection{Comparison}

From Figs. 13 and 14, it can be found that, the performance of active damping method by building $R_{v}$ is better 


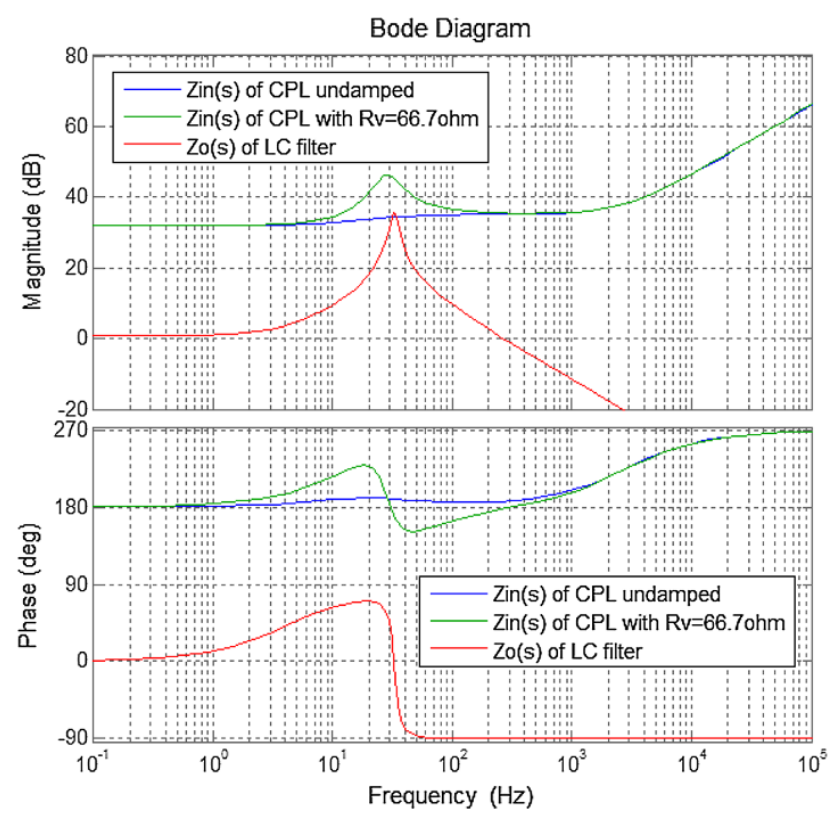

Fig. 11 The bode diagram of $Z_{o}(s)$ of LC filter and $Z_{\text {in }}(s)$ of CPL without damping method and with virtual resistor active damping method
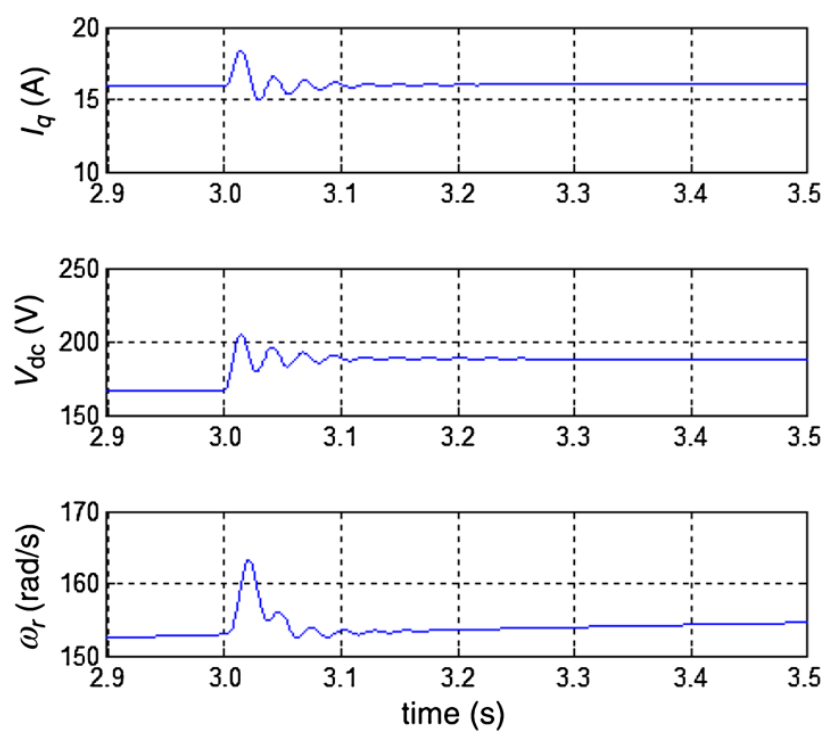

Fig. 12 The bode diagram of $Z_{o}(s)$ of LC filter and $Z_{\text {in }}(s)$ of CPL without damping method and with virtual capacitor active damping method

than the active damping method by building $C_{v}$, because the former method can achieve almost the same damping effect with relatively smaller undesirable oscillation in rotating speed.

Mathematically, the comparison can be made based on root locus method. Assume that these two methods are applied in an unstable cascaded system separately. Due to

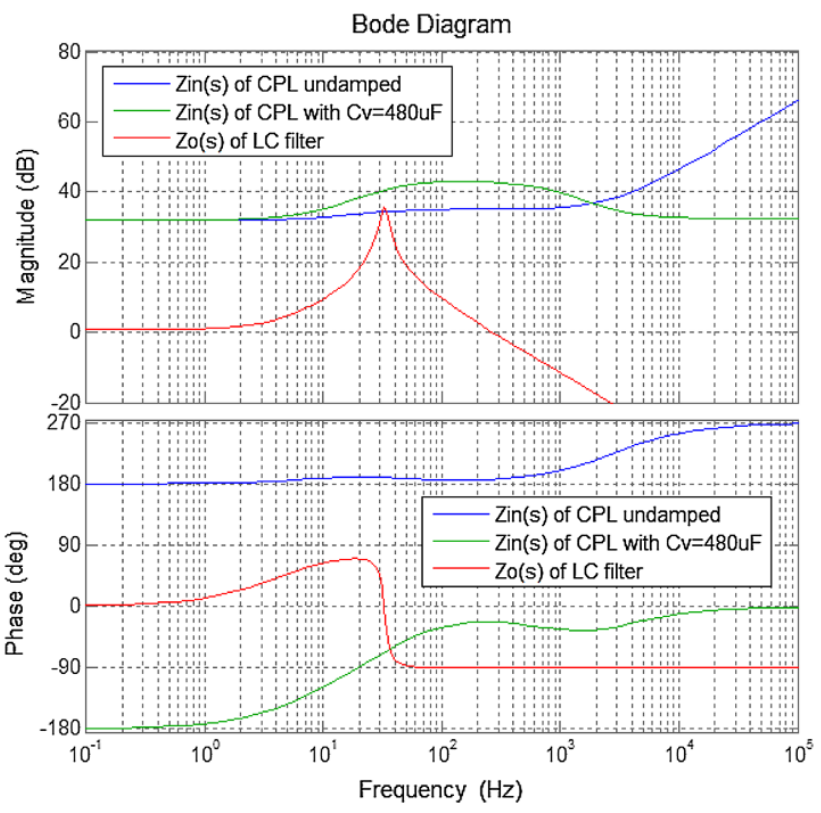

Fig. 13 The performances of a DC brushless motor as a CPL with active damping method by building virtual resistor $R_{v}=10 \Omega$
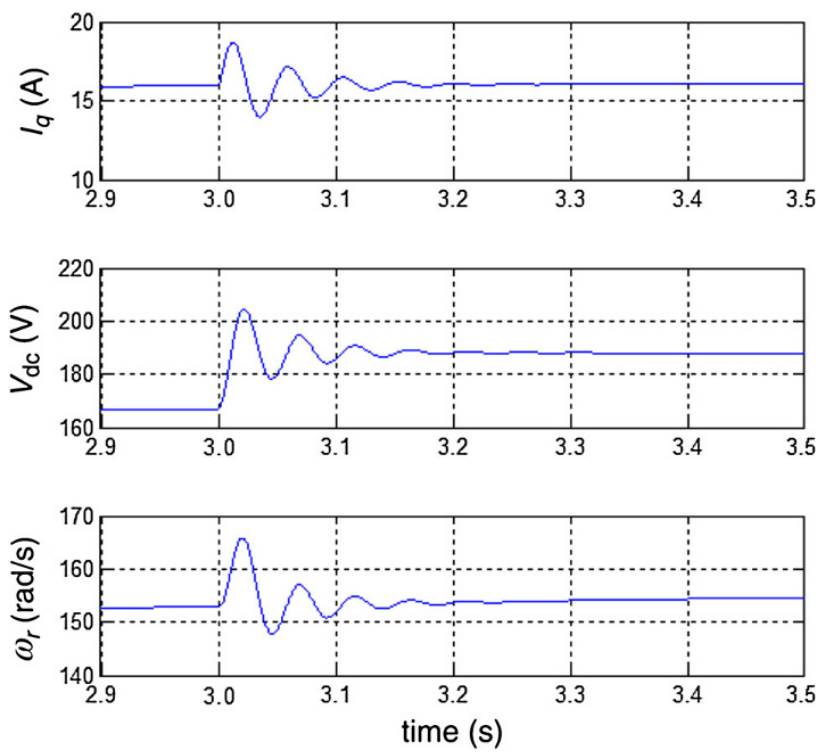

Fig. 14 The performances of a DC brushless motor as a CPL with active damping method by building virtual capacitor $C_{v}=1,200 \mu \mathrm{F}$

that both $C_{b}(s)$ and $C_{l}(s)$ have unity gain, the amplitude of $i_{\text {stab }}$ only depends on $1 / R_{v}$ in (13) or $\omega C_{v}$ in (14). We can compare these two methods by examining the damping effort with the same amplitude of $i_{\text {stab }}$. Thus, we assume that

$\frac{1}{R_{v}}=\omega C_{v}$

Therefore, the amplitude of $i_{\text {stab }}$ in these two methods are the same. In another words, the undesirable effects on 


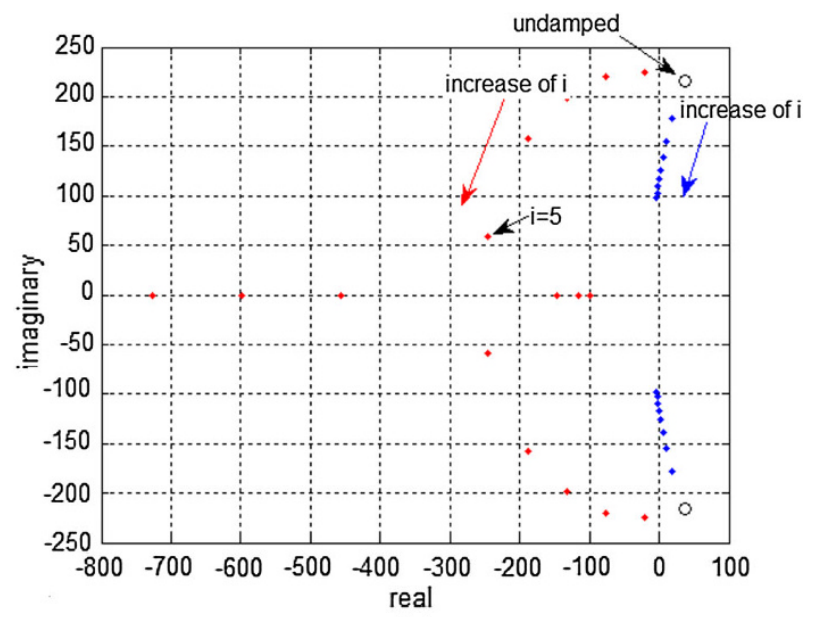

(a)

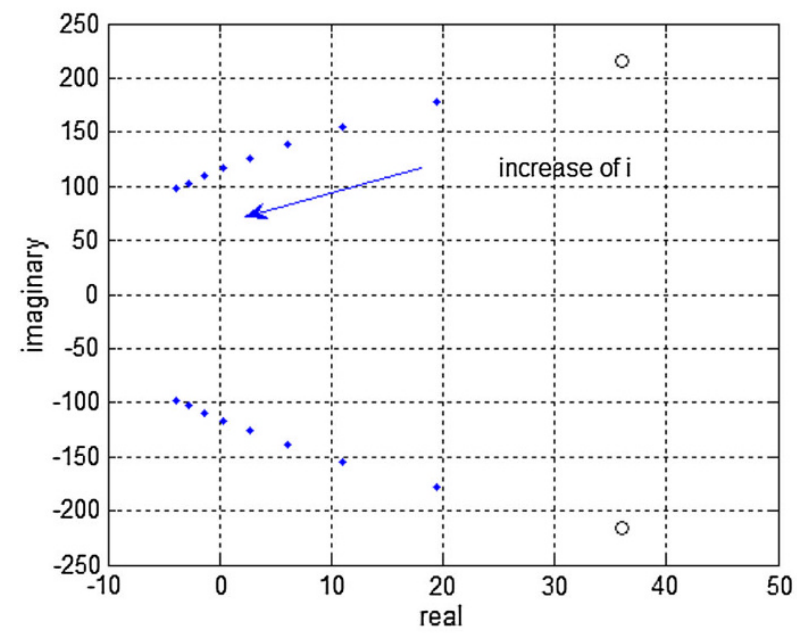

(b)

Fig. 15 a The root loci of the cascaded system with method of building virtual resistor $R_{v}$ (in red) and with method of building virtual capacitor $C_{v}$ (in blue) and $\mathbf{b}$ the details around zero

the load performance are the same. A series of $R_{v}$ and $C_{v}$ are selected as shown in (16) and (17).

$C_{v}=0.5 C i$

$R_{v}=\frac{1}{0.5 \omega C i}$

where $i=1,2, \ldots, 8$. The root loci of the cascaded system with two active methods are shown separately in Fig. 15.

In Fig. 15, it can be observed that when $i$ increases, the poles of the system with the method of building virtual resistor can approach to the negative real part much quicker comparing with the method of building virtual capacitor. Therefore, with the same amplitude of $i_{\text {stab }}$, i.e. the same undesirable effect on the load performance, the damping effort of the method of building virtual resistor is better than the method of building virtual capacitor.

\subsection{Nonlinear methods}

Sudhoff S. D. et al proposed a nonlinear method [28]. However, this method has limited damping effect compared with the linear method. This has been presented in [39]. A passivity based control method is proposed in [39]. This passivity based control can provide better damper without large undesirable load performance. The passivity based control algorithm is shown in (13) and (14).

$\theta=-R_{L} \frac{P}{V_{d c}}+R_{1}\left(i-\frac{P}{V_{d c}}\right)+V_{i n}$

$P_{\text {stab }}=u\left(\frac{P}{V_{d c}}-C \frac{\mathrm{d} \theta}{\mathrm{d} t}\right)-P+\frac{(u-\theta) u}{R_{2}}$

where $R_{1}$ and $R_{2}$ are two coefficients. $\theta$ and $V_{d c}$ are reference value of $i$ and $u$. However, complicated calculation with measurements $i, u$ and $P$ as shown (18) and (19) can result in large error and noises. As a result, this method is difficult to be implemented.

\subsection{Sensitivity and availability}

As shown in Fig. 9, a stabilizing power is injected into the CPL to stabilize the cascaded system. This stabilizing power is a transient oscillating component. It can result in undesirable load performances, such as the oscillation in the rotating speed of the motors. Therefore, there is always a compromise between the damping of the oscillation in LC input filter and the load performances. Therefore, sensitivity from input voltage of CPL to the rotating speed is important in the design of active stabilization method.

As the injected power is realized by the downstream converters, a large stabilizing power injected into the CPL is required to achieve a greater damping effect. This implies a wide range of duty cycle values in the downstream converters. However, the duty cycles are within the range of $(0,1)$. Therefore, the stabilizing effect is limited by available duty cycle range, e.g. high step-up converters usually operate at duty cycle between 0.5 and 1 . Thus the availability of the stabilizing effect of the active stabilization method is another aspect to be considered.

\section{Active damping method 3: adding a shunt impedance $Z_{a}(s)$}

Owing to the sensitivity and availability problems in the active stabilization in Section 3, a new type of methods are proposed. In this type of methods, an auxiliary DC/DC converter is added across the input voltage of the CPL. This DC/DC converter works as a power buffer and also 


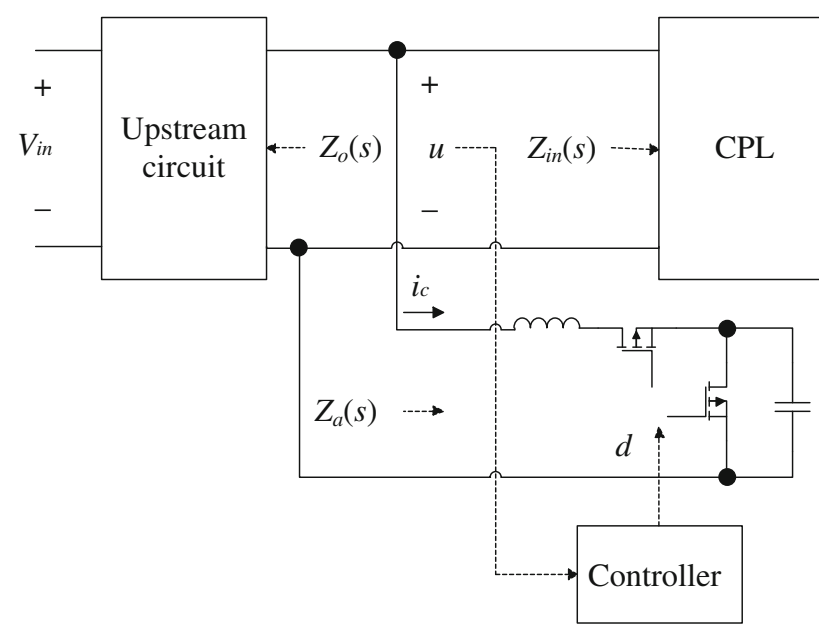

Fig. 16 The schematic diagram of the extra power electronic circuit

can be designed to decouple the interaction between the CPL and its LC input filter.

As shown in Fig. 16, an auxiliary power electronic circuit is added to stabilize the system. The input current $i_{c}$ is controlled depending on the input voltage $u$ to control its input impedance $Z_{a}(s)$. In order to fulfill the Middlebrook's stability, $Z_{a}(s)$ should fulfill

$\left\|Z_{o}(s)\right\|<\left\|\frac{Z_{a}(s) Z_{i n}(s)}{Z_{a}(s)+Z_{\text {in }}(s)}\right\|$

Or

$\left\|\frac{Z_{a}(s) Z_{o}(s)}{Z_{a}(s)+Z_{o}(s)}\right\|<\left\|Z_{i n}(s)\right\|$

In addition, $\left\|Z_{a}(s)\right\|$ should approach infinity when frequency is zero.

Theoretically, this auxiliary DC/DC converter can stabilize the system as the CPL does as described in Section 3 and it does not inherit the sensitivity problem. It can work as a virtual resistor or a virtual capacitor or use to realize nonlinear methods.

In [30], an auxiliary DC/DC converter is presented to mimic a virtual capacitor. This is realized by the control of the input current of the auxiliary DC/DC converter, $i_{c}$, according to the capacitor voltage $u$. i.e.

$i_{c}=C_{v} \frac{\mathrm{d} u}{\mathrm{~d} t}$

where $C_{v}$ is the virtual capacitor.

In [29], an auxiliary DC/DC converter is used to realize a pole placement method. In this method, the duty cycle of the auxiliary converter is controlled to replace the poles of the whole dynamic system. The capacitor voltage, $u$, and the input current of the auxiliary converter, $i_{c}$, are measured and second order observer is used. Thus, all four system states, inductor current of LC filter, $i$, capacitor voltage of the auxiliary converter, $v_{c}, i_{c}$ and $u$ are available for controller design. Therefore, the poles of the closed loop control system can be replaced to obtain a good damping performance.

\section{Comparison and conclusion}

This paper analyzes several existing active stabilization methods. The methods have been evaluated through Middlebrook's stability criterion. According to the source of the stabilizing effect, these methods are classified into three categories. The advantages and disadvantages of these three types of methods are summarized as follows:

The active damping method which reduces the output impedance of the upstream converter is discussed in Section 2. The main advantage of this type of methods is that it can stabilize the cascaded system without affecting the operation and performance of CPL. The disadvantage of these methods is that the upstream circuit needs to be controllable, e.g. a switching regulator. These methods are suitable on the systems in which the feeder of CPLs is another stage converter.

The active damping method which increases the input impedance of the CPL is discussed in Section 3. The main merit is that the CPL can overcome the negative impedance instability by itself. These methods are suitable for the systems in which the feeder of CPL is an uncontrollable LC filter. The demerit of these methods is that the stabilizing power injected into the CPL can result in some undesirable load performances. There is always a compromise between the damping effect and the load performances.

The active damping methods by adding a shunt impedance through an extra power electronics circuit is discussed in Section 4. The strength of this method is that the extra power electronic converter can stabilize the system without the undesirable effect on the load performances. This type of methods has a high potential to achieve similar function as the CPL does to stabilize the system, but without the compromise. The weak side is that extra circuit is required resulting in additional cost and power losses.

Open Access This article is distributed under the terms of the Creative Commons Attribution License which permits any use, distribution, and reproduction in any medium, provided the original author(s) and the source are credited.

\section{References}

[1] Rahimin AM, Khaligh A, Emadi A (2006) Design and implementation of an analog constant power load for studying cascaded converters. In: Proceedings of the 32nd annual conference 
on IEEE industrial electronics society (IECON'06), Paris, 6-10 Nov 2006, pp 1709-1714

[2] Liu XY, Forsyth AJ, Cross AM (2007) Negative input-resistance compensator for a constant power load. IEEE Trans Ind Electron 54(6):3188-3196

[3] Kwasinski A, Krein PT (2007) Passivity-based control of buck converters with constant-power loads. In:Proceedings of the 2007 IEEE power electronics specialists conference (PESC'07), Orlando, 17-21 Jun 2007, pp 259-265

[4] Emadi A, Khaligh A, Rivetta CH et al (2006) Constant power loads and negative impedance instability in automotive systems: Definition, modeling, stability, and control of power electronic converters and motor drives. IEEE Trans Veh Technol 55(4):1112-1125

[5] Rivetta C, Williamson GA (2004) Global behaviour analysis of a DC-DC boost power converter operating with constant power load. In: Proceedings of the 2004 international symposium on circuits and systems (ISCAS'04), vol 5. Vancouver, Canada, 23-26 May 2004, pp 956-959

[6] Rivetta CH, Emadi A, Williamson GA et al (2006) Analysis and control of a buck DC-DC converter operating with constant power load in sea and undersea vehicles. IEEE Trans Ind Appl 42(2):559-572

[7] Rahimi AM, Emadi A (2009) An analytical investigation of DC/DC power electronic converters with constant power loads in vehicular power systems. IEEE Trans Veh Technol 58(6):2689-2702

[8] Riccobono A, Santi E (2012) Comprehensive review of stability criteria for DC power distribution systems. In: Proceedings of the 2012 IEEE energy conversion congress and exposition (ECCE'12), Raleigh, 15-20 Sept 2012, pp 3917-3925

[9] Middlebrook RD (1976) Input filter considerations in design and application of switching regulators. In: Proceedings of the IEEE industry applications society annul meeting (IAS'76), Chicago, 11-14 Oct 1976, pp 366-382

[10] Wildrick CM, Lee FC, Cho BH et al (1995) A method of defining the load impedance specification for a stable distributed power system. IEEE Trans Power Electron 10(3):280-285

[11] Sudhoff SD, Glover SF, Lamm PT et al (2000) Admittance space stability analysis of power electronic systems. IEEE trans aero electron syst 36(3-Part 1):965-973

[12] Feng XG, Liu JJ, Lee FC (2002) Impedance specifications for stable DC distributed power systems. IEEE Trans Power Electron 17(2):157-162

[13] Du W, Zhang J, Zhang Y et al (2013) Stability criterion for cascaded system with constant power load. IEEE Trans Power Electron 28(4):1843-1851

[14] Rahimi AM, Emadi A (2009) Active damping in DC/DC power electronic converters: A novel method to overcome the problems of constant power loads. IEEE Trans Ind Electron 56(5):1428-1439

[15] Rahimi AM, Williamson GA, Emadi A (2010) Loop-cancellation technique: A novel nonlinear feedback to overcome the destabilizing effect of constant-power loads. IEEE Trans Veh Technol 59(2):650-661

[16] Onwuchekwa CN, Kwasinski A (2009) Boundary control of buck converters with constant-power loads. In: Proceedings of the 31st international telecommunications energy conference (INTELEC'09), Incheon, 18-22 Oct 2009, 6 pp

[17] Onwuchekwa CN, Kwasinski A (2011) Analysis of boundary control for boost and buck-boost converters in distributed power architectures with constant-power loads. In: Proceedings of the 26th annual IEEE applied power electronics conference and exposition (APEC'11), Fort Worth, 6-11 Mar 2011, pp 1816-1823

[18] Zhao Y, Qiao W, Ha D (2014) A sliding-mode duty-ratio controller for DC/DC buck converters with constant power loads. IEEE Trans Ind Appl 50(2):1448-1458

[19] Zeng J, Zhang Z, Qiao W (2014) An interconnection and damping assignment passivity-based controller for a DC-DC boost converter with a constant power load. IEEE Trans Ind Appl 50(4):2314-2322

[20] Radwan AAA, Mohamed YARI (2012) Linear active stabilization of converter-dominated DC microgrids. IEEE Trans Smart Grid 3(1):203-216

[21] Zhang XN, Vilathgamuwa DM, Foo G, et al (2013) Cascaded sliding mode control for global stability of three phase AC/DC PWM rectifier with rapidly varying power electronic loads. In: Proceedings of the 39th annual conference of the IEEE industrial electronics society, (IECON'13), Vienna, 10-13 Nov 2013, pp 4580-4587

22. Magne P, Marx D, Nahid-Mobarakeh B et al (2012) Large-signal stabilization of a DC-link supplying a constant power load using a virtual capacitor: Impact on the domain of attraction. IEEE Trans Ind Appl 48(3):878-887

[23] Mohamed YARI, Radwan AAA, Lee TK (2012) Decoupled reference-voltage-based active DC-link stabilization for PMSM drives with tight-speed regulation. IEEE Trans Ind Electron 59(12):4523-4536

[24] Lee WJ, Sul SK (2014) DC-link voltage stabilization for reduced DC-link capacitor inverter. IEEE Trans Ind Appl 50(1):404-414

[25] Liutanakul P, Awan AB, Pierfederici S et al (2010) Linear stabilization of a DC bus supplying a constant power load: A general design approach. IEEE Trans Power Electron 25(2): $475-488$

[26] Glover SF, Sudhoff SD (1998) An experimental validated nonlinear stabilizing control for power electronics based power systems. In: Proceedings of the SAE aerospace power system conference (APSC'98), Detroit, 21-23 Apr 1998, pp 71-88

[27] Mosskull H, Galic J, Wahlberg B (2007) Stabilization of induction motor drives with poorly damped input filters. IEEE Trans Ind Electron 54(5):2724-2734

[28] Sudhoff SD, Corzine KA, Glover SF et al (1998) DC link stabilized field oriented control of electric propulsion system. IEEE Trans Energ Conver 13(1):27-33

[29] Inoue K, Kato T, Inoue M, et al (2012) An oscillation suppression method of a DC power supply system with a constant power load and a LC filter. In: Proceedings of the IEEE 13th workshop on control and modeling for power electronics (COMPEL'12), Kyoto, 10-13 Jun 2012, 4 pp

[30] Zhang X, Ruan X, Kim H et al (2013) Adaptive active capacitor converter for improving stability of cascaded DC power supply system. IEEE Trans Power Electron 28(4):1807-1816

[31] Grigore V, Hatonen J, Kyyra J et al (1998) Dynamics of a buck converter with a constant power load. In: Proceedings of the 29th annual IEEE power electronics specialists conference (PESC'98), vol 1. Fukuoka, 17-22 May 1998, pp 72-78

[32] Rahimi AM, Emadi A (2010) Discontinuous-conduction mode DC/DC converters feeding constant-power loads. IEEE Trans Ind Electron 57(4):1318-1329

[33] Spiazzi G, Mattavelli P, Rossetto L (1997) Power factor preregulators with improved dynamic response. IEEE Trans Power Electron 12(2):343-349

[34] Prodic A, Chen JQ, Maksimovic D, et al (2003) Self-tuning digitally controlled low-harmonic rectifier having fast dynamic response. IEEE trans power electron 18(1-Part 2):420-428

[35] Lamar DG, Fernandez A, Arias M et al (2008) A unity power factor correction preregulator with fast dynamic response based on a low-cost microcontroller. IEEE Trans Power Electron 23(2):635-642

[36] Wall S, Jackson R (1997) Fast controller design for single-phase power-factor correction systems. IEEE Trans Ind Electron 44(5):654-660

[37] Rezaei K, Golbon N, Moschopoulos G(2014) A new control scheme for an AC-DC single-stage buck-boost PFC converter 
with improved output ripple reduction and transient response. In: Proceedings of the 29th annual IEEE applied power electronics conference and exposition (APEC'14), Fort Worth, 16-20 Mar 2014, pp 1866-1873

[38] Belkhayat M, Cooley R, Witulski A (1995) Large signal stability criteria for distributed systems with constant power loads. In: Proceedings of the 26th annual IEEE power electronics specialists conference (PESC '95), vol 2. Atlanta, 18-22 Jun 1995, pp $1333-1338$

[39] Liu XY, Forsyth AJ (2005) Comparative study of stabilizing controllers for brushless DC motor drive systems. In: Proceedings of the 2005 IEEE international conference on electric machines and drives, San Antonio, 15-18 May 2005, pp 1725-1731

Mingfei WU received the B.Eng. degree in Electrical and Electronic Engineering from Northumbria University, UK, and M.Sc. degree in Control Systems from Imperial College London, UK, in 2009 and 2010, respectively. From 2012, he started to pursue his Ph.D. degree in the University of Sydney, Australia. His current research focuses on the stability of DC microgrids.
Dylan DAH-CHUAN LU received his Ph.D. degree in Electronic and Information Engineering from The Hong Kong Polytechnic University, Hong Kong, China, in 2004. In 2003, he joined PowereLab Ltd. as a Senior Engineer. His major responsibilities include project development and management, circuit design, and contribution of research in the area of power electronics. In 2006, he joined the School of Electrical and Information Engineering, The University of Sydney, Australia, where he is currently a Senior Lecturer. He presently serves as an Associate Editor of the International Journal of Electronics, and the Australian Journal of Electrical and Electronics Engineering. He is an author/co-author of over 90 technical articles in the areas of power electronics and engineering education. He has two patents on efficient power conversion. His current research interests include power electronics circuits and control for efficient power conversion, lighting, renewable electrical energy systems, microgrid and power quality improvement, and engineering education. Dr. LU received the Dean's Research Award in 2011. He is a senior member of the IEEE and a member of the IEAust. 